\title{
AURICULOTERAPIA COMO TRATAMENTO COMPLEMENTAR À FISIOTERAPIA NA QUALIDADE DE VIDA EM IDOSOS COM INCONTINÊNCIA URINÁRIA
}

\author{
AURICULOTHERAPY AS COMPLEMENTARY TREATMENT TO \\ PHYSICAL THERAPY IN THE QUALITY OF LIFE OF THE ELDERY \\ WITH URINARY INCONTINENCE
}

\author{
Martinez, A.B. ${ }^{1}$, Ribeiro, S.S. ${ }^{1}$, Samagaia, G.C.Z. ${ }^{1}$, Valente, C. ${ }^{1}$ \\ Afiliações: 1- Centro de Ciências da Saúde da Universidade Regional de Blumenau - FURB. \\ Endereço do autor de correspondência: Rua Pará, 881, Industrial, Francisco Beltrão, Paraná, Brasil. CEP: 85601-290. \\ carolvalente11@gmail.com.
}

\section{Resumo}

Incontinência Urinária é a perda involuntária de urina, que afeta de maneira negativa a qualidade de vida das pessoas, causando-as desconfortos para sair de casa, depressão, baixa autoestima e interfere até em seu psicológico. Com isso, a fisioterapia apresenta métodos capazes de promover a diminuição da perda urinária, interferindo positivamente na qualidade de vida. A auriculoterapia também é uma prática integrativa, que quando aplicada corretamente, apresenta melhora dessa disfunção. O presente trabalho tem como objetivo avaliar a eficácia da auriculoterapia na qualidade de vida de idosos com incontinência urinaria (IU) como tratamento complementar à fisioterapia. Trata-se de uma pesquisa descritiva e transversal, realizada com três idosos com idade superior a 60 anos. Foram utilizados como instrumento desta pesquisa dois questionários, o King's Health Questionnaire (KHQ) traduzido e adaptado para o português, e o International Consutation on Incontinence Questionnaire - Short Form (ICIQ-SF) para avaliar o impacto da IU na qualidade de vida. Como tratamento, foram realizados exercícios fisioterapêuticos nos três idosos, e em dois deles foi aplicada a auriculoterapia para auxiliar na redução de perda urinária. Com base nos questionários aplicados, a diferença dos resultados do início do atendimento para o final do atendimento não teve uma diferença significativa. Porém, de acordo com os relatos dos pacientes, eles notaram a diminuição da perda urinária e também o aumento do tempo de controlar a urina.

Palavras-chave: Auriculoterapia; Incontinência urinária; Fisioterapia; Qualidade de vida; Idosos.

\section{Abstract}

Urinary Incontinence is the involuntary loss of urine, which adversely affects people's quality of life, causing them discomfort to leave the home, depression, low self-esteem and even interferes with their psychological. With this, physiotherapy presents methods capable of promoting the decrease of urinary loss, interfering positively in the quality of life. Auriculotherapy is also an integrative practice, which, when applied correctly, improves this dysfunction. The present study aims to evaluate the effectiveness of auriculotherapy in the quality of life of the elderly with urinary incontinence (UI) as a complementary treatment to physical therapy. This is a descriptive and cross-sectional study carried out with three elderly individuals over 60 years. Two questionnaires, the King's Health Questionnaire (KHQ) translated and adapted to Portuguese, and the International Consutation on Incontinence Questionnaire - Short Form (ICIQ-SF) were used to evaluate the impact of UI on quality of life. As a treatment, physiotherapeutic exercises were performed in the three elderly patients, and two of them were applied auriculotherapy to help reduce urinary loss. Based on the questionnaires applied, the difference in the results from the beginning of care to the end of care did not have a significant difference. However, according to the patients' reports, they noted a decrease in urinary loss and also an increase in the time to control urine.

Keywords: Auriculotherapy; Urinary Incontinence; Physical Therapy Specialty; Quality of Life; Aged. 


\section{Introdução}

As disfunções do assoalho pélvico são causadas por alterações em ligamentos, fáscias e na musculatura do assoalho pélvico. A incontinência urinária, considerada uma das disfunções do Assoalho Pélvico (AP), ocorre devido à diminuição da capacidade de suporte da musculatura do assoalho pélvico (MAP) ou por problemas vesicais, os sintomas que eles acarretam não ameaçam a vida do paciente, porém afetam de um jeito negativo a sua qualidade de vida interferindo em suas relações sociais, em suas atividades físicas e até em seu psicológico ${ }^{1}$.

Incontinência urinária (IU) é definida como qualquer perda involuntária de urina objetivamente demonstrável que tenha repercussão social, acarretando problemas de saúde adicionais e, por conseguinte, impactando negativamente na qualidade de vida ${ }^{2}$. Há três tipos de IU: incontinência urinária de esforço, incontinência urinária de urgência e incontinência urinária mista.

Incontinência urinária de esforço (IUE)

é definida como perda involuntária de urina durante esforço, prática de exercício, ao tossir ou espirrar $^{3}$, causada pela hipermobilidade uretral e pelo comprometimento do esfíncter uretral. A incontinência urinária de urgência se caracteriza pelo fato da perda involuntária de urina aparecer precedida da necessidade urgente de urinar, causada pelas contrações involuntárias do músculo detrusor (hiperatividade vesical), que aumenta a pressão intravesical ${ }^{3}$. Quando existe associação dos sintomas de incontinência de esforço e de urgência, classifica-se incontinência urinária mista.

A fisioterapia apresenta uma grande importância no tratamento dessa patologia. Existem diversos métodos, sendo eles invasivos como os cones vaginais, perineômetro, biofeedback e eletroestimulação que servem para realizar o fortalecimento da musculatura perineal e melhora da percepção da musculatura utilizada. Os métodos não invasivos são a estimulação magnética perineal utilizada para gerar contração da musculatura, a reeducação comportamental para estipular o ritmo miccional e a cinesioterapia para melhorar a resistência e força da musculatura do assoalho pélvico através de exercícios realizados pelo paciente.

A auriculoterapia é um método terapêutico que utiliza a orelha para diagnóstico e tratamento de diversas enfermidades através da utilização de agulhas, sementes ou cristais. Cada ponto do nosso corpo possui uma 
correspondência auricular que se torna perceptível quando uma região do corpo perde seu equilíbrio fisiológico. O pavilhão auricular é ligado a diversas áreas do corpo através dos canais de energia e do sistema nervoso ${ }^{4}$.

Com base nisso, optamos por complementar a fisioterapia com auriculoterapia, visto que essa prática alternativa apresenta benefícios fisiológicos e ajudaria a melhorar a qualidade de vida dos idosos com incontinência urinária, utilizando pontos que estimulam a sustentação dos músculos para que eles consigam controlar a perda; ponto para controlar a ansiedade e diminuir a tensão muscular. Também foram utilizados pontos para o sistema urinário, tranquilizante da mente e melhorar a função do fígado, vesícula biliar e estômago.

Esse estudo tem por objetivo avaliar a eficácia da auriculoterapia na qualidade de vida de idosos com incontinência urinária como tratamento complementar à fisioterapia uroginecológica, realizando, para isso, atendimentos de cinesioterapia e cinesioterapia com auriculoterapia, de modo a comparar os resultados.

\section{Métodos}

A pesquisa é descritiva e transversal, buscou analisar os efeitos da auriculoterapia como terapia complementar no tratamento de incontinência urinária, juntamente com atendimentos de fisioterapia uroginecológica. Através da análise quantitativa e qualitativa, buscou-se levantar dados para estabelecer a melhora na qualidade de vida de idosos com incontinência.

O universo de pesquisa foram os pacientes atendidos pelo Centro de Saúde do Idoso, vinculado à Secretaria Municipal de Promoção de Saúde da Prefeitura Municipal de Blumenau, localizada na rua Itapiranga $n^{\circ} 368$, bairro da Velha. A estrutura oferece atenção integral à saúde do idoso, através da reabilitação ampla ao idoso frágil com os profissionais de fisioterapia, medicina, enfermagem, odontologia, psicologia, terapia ocupacional, fonoaudiologia, nutrição e assistente social.

Os pacientes da pesquisa foram idosos de ambos os sexos, inseridos no Centro de Saúde do Idoso, de idade igual ou superior a 60 anos, com queixas de perda involuntária de urina e insatisfação com sua qualidade de vida devido a essa perda. Os critérios de exclusão foram déficits cognitivos graves e estar realizando tratamento fisioterapêutico para IU e prolapsos. 
Foi considerado como critério de descontinuidade a não realização completa da avaliação inicial e não realização das sessões agendadas.

Na avaliação da pesquisa será utilizado o King's Health Questionnaire (KHQ) traduzido e adaptado para o português. É composto por trinta perguntas que são arranjadas em nove domínios. Relatam, respectivamente, a percepção da saúde, o impacto da incontinência, as limitações do desempenho das tarefas, a limitação física, a limitação social, o relacionamento pessoal, as emoções, o sono e a energia e as medidas de gravidade. Existe também uma escala de sintomas que é composta pelos seguintes itens: frequência urinária, noctúria, urgência, hiperreflexia vesical, incontinência urinária de esforço, enurese noturna, incontinência no intercurso sexual, infecções urinárias e dor na bexiga. Há, também, um espaço para os pacientes relatarem qualquer outro problema que possam ter relacionado com a bexiga 5 .

\section{O International Consultation on} Incontinence Questionnaire - Short Form (ICIQSF) é um questionário auto-administrável que avalia o impacto da IU na qualidade de vida e a qualificação da perda urinária dos pacientes analisados. O ICIQ-SF é composto de quatro questões que avaliam a frequência, a gravidade e o impacto da IU, além de um conjunto de oito itens de autodiagnóstico, relacionados às causas ou a situações de IU vivenciadas pelos pacientes ${ }^{6}$.

Para auriculoterapia será aplicado a auriculoterapia francesa, sendo esta forma de tratamento não invasiva, utilizando 10 pontos, sendo eles: Triângulo Cibernético (Shem Men, Rim, Tronco Nervoso Cerebral), Bexiga, Baço, Fígado, Vesícula Biliar, Ansiedade, Tensão e Sanjiao. O material para aplicação é semente de mostarda coberta por esparadrapo hipoalergênico na cor bege, para limpeza do local de aplicação utilizaremos álcool 70\% e algodão hidrófilo.

Foram selecionados três participantes, separados em dois grupos: o Grupo Auriculoterapia (grupo A) com dois pacientes que receberam 7 atendimentos de fisioterapia e auriculoterapia em 4 semanas; e o Grupo Controle (grupo B) com um paciente que realizou 7 atendimentos de fisioterapia em 4 semana sem auriculoterapia.

Os questionários foram aplicados de forma independente, onde os pacientes ficaram sozinhos em uma sala silenciosa para poder 
responder de maneira mais confortável e sem que houvesse barulhos para desconcentrá-los.

No início dos atendimentos, era aplicada a auriculoterapia nos dois pacientes, e na sequencia realizados os exercícios fisioterapêuticos. Os exercícios de cinesioterapia foram aplicados igualmente em todos os pacientes.

Eles constituíam de fortalecimento da MAP, pedindo para que o paciente realizasse a contração dessa musculatura, como se estivesse "segurando o xixi". Fortalecimento de membros inferiores (MMII) era realizado com resistência de thera band para trabalhar com os abdutores do quadril, exercícios de apertar a bola no meio dos MMII para os adutores, e exercício de ponte para trabalhar glúteos.

A mobilização pélvica era realizada com o paciente deitado com os MMII apoiados sobre a bola, onde eram executados os movimentos de extensão, flexão, rotações internas e externas de quadril. No final do atendimento era realizado alongamento de adutores de quadril.

\section{Resultados}

O trabalho foi realizado com três pacientes no total, sendo eles dois do sexo feminino e um do sexo masculino. No primeiro atendimento foram aplicados os dois questionários de qualidade de vida sobre incontinência urinária. No questionário King's Health Questionnaire sugerimos valores de referência para cada item dos nove domínios aplicados, sendo eles valor 4 para "muito boa"; valor 3 para "boa / muito / sempre"; valor 2 para "normal / mais ou menos / várias vezes"; valor 1 para "ruim / um pouco / ás vezes"; e valor 0 para "muito ruim / não / nenhuma".

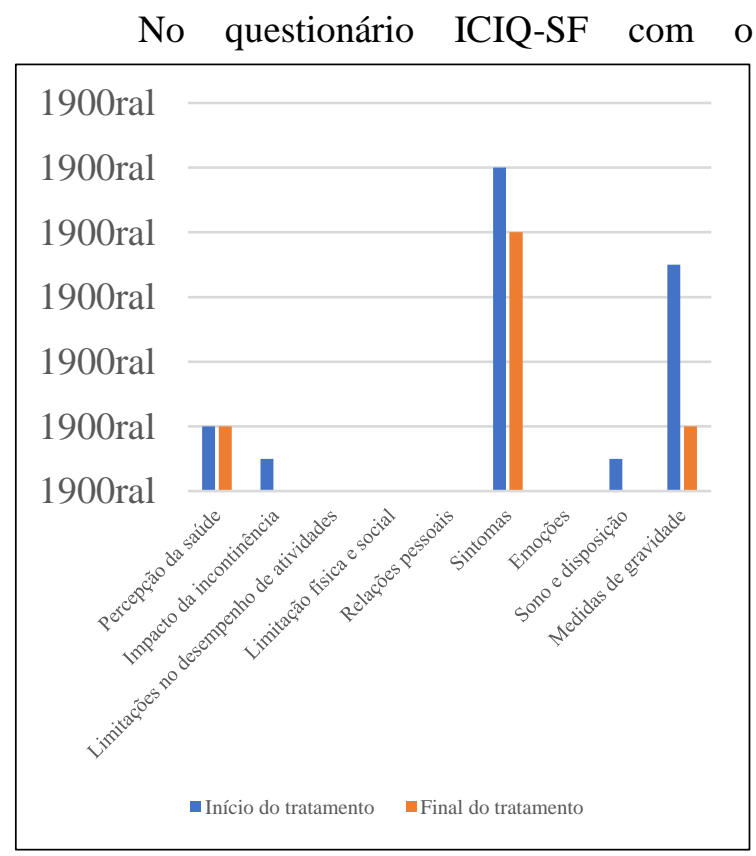

Figura 1. Dados do paciente 1 no King's Health Quesionnaire (KHQ)

paciente 1 , no início do tratamento, obtivemos resultado de 14 , indicando impacto muito grave em sua qualidade de vida, devido aos escapes urinários. E no final do tratamento o resultado obtido foi 3, indicando impacto leve. 


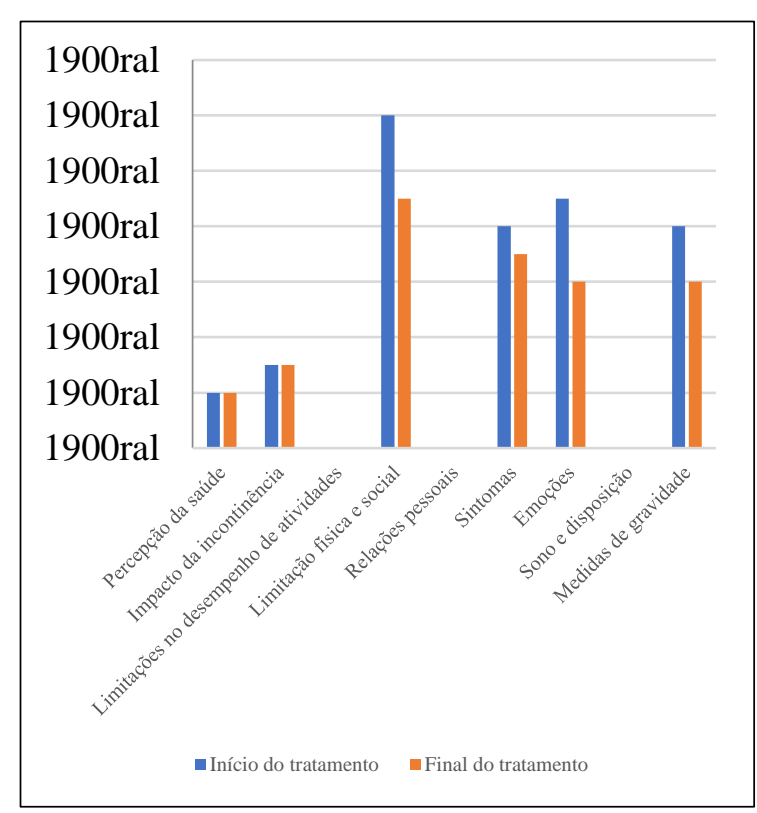

Figura 2. Dados do paciente 2 no King's Health Questionnaire (KHQ).

No paciente 2, no inicio do tratamento,

obtivemos o resultado 20 com o questionário ICIQ-SF, indicando impacto muito grave em sua qualidade de vida, devido aos escapes urinários. Ao final do tratamento o resultado foi 18 , ainda indicando impacto muito grave.

Com o paciente 3, no início do tratamento, obtivemos o resultado 11 no questionário ICIQ-SF, indicando impacto muito grave em sua qualidade de vida, devido aos escapes urinários. Ao final do tratamento o resultado foi 9, indicando um impacto grave em sua qualidade de vida, devido aos escapes urinários.

\section{Discussão}

$\mathrm{O}$ paciente 1, realizou apenas os exercícios de fisioterapia uroginecológica, apresentou evolução de 11 pontos no questionário ICIQ-SF. É uma melhora considerável, e perceptível pelo paciente, que relatou, ao final do último atendimento:

\begin{abstract}
"O xixi, antes nas atividades, eu não conseguia aguentar tanto tempo. Às vezes eu chegava o banheiro e já dava um pingo, e agora não. Agora tranquilo, só desce uma gotinha quando eu espirro e estou muito apurada, se não também não”.
\end{abstract}

No questionário KHQ, o paciente 1 apresentou melhora considerável em medidas de gravidade, e alguma melhora em sono e disposição, sintomas e impacto na incontinência, mantendo estável a avaliação de percepção de saúde. As demais variáveis do questionário KHQ não se encaixaram ao paciente.

$\mathrm{O}$ paciente 2 realizou os atendimentos em fisioterapia uroginecológica e auriculoterapia, e apresentou uma evolução de 2 pontos no questionário ICIQ-SF, que ainda indicou impacto muito grave. Contudo, o paciente relatou uma melhora perceptível: "Eu percebi que não acordava mais com a cama molhada durante a noite", bem como sua cuidadora, cuidadora que notou que as fraldas estavam menos cheias de urina. 
No questionário KHQ, o paciente 2 apresentou melhora nos aspectos de limitação física e social, sintomas, emoções e medidas de gravidade, mantendo estável a autoavaliação em percepção da saúde e impacto na incontinência. Nas dimensões "limitações no desempenho de tarefas", "relações pessoais" e "sono e energia", nenhuma alternativa se aplicou ao paciente, não entrando na somatória.

O paciente 3 recebeu os atendimentos de fisioterapia uroginecológica e auriculoterapia, e apresentou, no questionário ICIQ-SF, uma melhora de 2 pontos. O paciente relatou melhoras:

\footnotetext{
"Percebi que a queimação que eu sentia em minha bexiga diminuiu, e também notei que consigo segurar o xixi até chegar ao banheiro com mais facilidade".
}

No questionário KHQ, o paciente apresentou grande pontuação nos critérios sintomas e medidas de gravidade. Na dimensão de relações pessoais, nenhuma alternativa se aplicou ao paciente, não entrando na somatória. Entretanto, o questionário não foi aplicado ao paciente 3 no final do atendimento, pois o mesmo não compareceu e não conseguimos fazer contato para realizar a aplicação.

Com esses resultados notamos que, apesar de não haver diferença significativa dos valores, houve melhora de alguns domínios como, por exemplo, "medidas de gravidade" no paciente 1; "emoções" e "limitação física e social", no paciente 2. De acordo com seus relatos, eles notaram a diminuição da perda urinária e também o aumento do tempo de controlar a urina.

\section{Conclusão}

O desenvolvimento do presente trabalho possibilitou uma análise da importância da fisioterapia para IU, e associada com a auriculoterapia a diminuição da perda urinária pôde ser notada em pouco tempo, consequentemente melhorando a qualidade de vida dos idosos. Apesar das dificuldades presentes na pesquisa, como o baixo número de participantes, a quantidade de atendimentos reduzidos e os resultados mostrarem valores pouco significativos, o diálogo com os pacientes constatou alterações positivas em suas vidas.

O trabalho apresentado mostrou a eficácia da auriculoterapia como tratamento complementar à fisioterapia na qualidade de vida de pacientes com IU, podendo divulgar essa prática da medicina tradicional chinesa (MTC) que não possui tanta visibilidade no nosso meio.

$\mathrm{O}$ fato de haver afinidade com o tema levou à elaboração deste trabalho, visto que 
proporcionou experiências na área. órgãos pélvicos. In: EDITORS.Manual de Proporcionou também conhecimento e Ginecologia. Lisboa(PT): Permanyer; 2011. v.2. satisfação por acompanhar a evolução e melhora p. 283-98.

da qualidade de vida dos pacientes, com a intervenção da fisioterapia e auriculoterapia.

Dada a importância ao assunto, torna-se necessário o desenvolvimento de pesquisas com a mesma temática, porém com maior número de participantes, atendimentos de fisioterapia e aplicação de auriculoterapia, para que haja diferença na hora de comparar os resultados iniciais com os finais.

\section{Referências}

1. Camillato ES, Barra AA, Junior ALS. Incontinence Questionnaire - Short Form" Incontinência urinária de esforço: fisioterapia (ICIQ-SF). Rev. Saúde Pública. 2004;38(3):428versus tratamento cirúrgico. Feminina. Jul 2012. 44. 40(4):187-94.

2. Sacomani ARS et al. Incontinência Urinária. In: Nardi AC et al, editors. Diretrizes Urologia AMB. ed. São Paulo (BR): [s.n.], 2014. cap. 8, p. 82-90.

3. Mascarenhas T. Disfunções do pavimento 4. Jóji, E. Auriculoterapia oriental: método Enomoto. 1. ed. São Paulo: Ícone; 2005.

5. Fonseca ESM, Camargo ALM, Castro RA, Sartori MGF, Fonseca MCM, Lima, GR, et al. Validação do questionário de qualidade de vida (King's Health Questionnaire) em mulheres brasileiras com incontinência urinária. Rev Bras Ginecol Obstet. 2005;27(5):235-42.

6. Tamanini JTN, Dambros M, D'Ancona CAL, Palma PCR, Júnior NRN. Validação para o português do "International Consultation on pélvico: incontinência urinária e prolapso dos 